\title{
Explicit Bound for the Prime Ideal Theorem in Residue Classes
}

\author{
Maciej Grześkowiak * \\ Adam Mickiewicz University, \\ Faculty of Mathematics and Computer Science, \\ Umultowska 87, 61-614 Poznań, Poland \\ maciejg@amu.edu.pl
}

\begin{abstract}
We give explicit numerical estimates for the generalized Chebyshev functions. Explicit results of this kind are useful for estimating of computational complexity of algorithms which generates special primes. Such primes are needed to construct an elliptic curve over prime field using complex multiplication method.
\end{abstract}

\section{Introduction}

Let $K$ denote any fixed totally imaginary field of the discriminant $\Delta=\Delta(K)$ and degree $[K: \mathbb{Q}]=2 r_{2}$, where $2 r_{2}$ is the number of complex-conjugate fields of $K$. Denote by $\mathfrak{f}$ a given non-zero integral ideal of the ring of algebraic integers $\mathcal{O}_{K}$ and by $H(\bmod \mathfrak{f})$ any ideal class $\bmod \mathfrak{f}$ in the "narrow" sense. Let $h_{\mathfrak{f}}^{*}(K)$ be the number of elements of $H$. Let $\chi(H)$ be a character of the abelian group of ideal classes $H(\bmod \mathfrak{f})$, and let $\chi(\mathfrak{a})$ be the usual extension of $\chi(H)$. Let $s=\sigma+i t$. The Hecke-Landau zeta-functions associated to $\chi$, are defined by

$$
\zeta(s, \chi)=\sum_{\mathfrak{a} \in \mathcal{O}_{K}} \frac{\chi(\mathfrak{a})}{(N \mathfrak{a})^{s}}, \quad \sigma>1,
$$

where $\mathfrak{a}$ runs through integral ideals and $N \mathfrak{a}$ is the norm of $\mathfrak{a}$. Throughout, $\chi_{0}$ denote the principal character modulo $\mathfrak{f}$. Let

$$
E_{0}=E_{0}(\chi)=\left\{\begin{array}{l}
1 \text { for } \chi=\chi_{0} \\
0 \text { for } \chi \neq \chi_{0}
\end{array}\right.
$$

If $\chi$ is a primitive character, then $\zeta(s, \chi)$ satisfies the functional equation

$$
\Phi(s, \chi)=W(\chi) \Phi(1-s, \bar{\chi}), \quad|W(\chi)|=1,
$$

where

$$
\Phi(s, \chi)=A(\mathfrak{f})^{s} \Gamma(s)^{r_{2}} \zeta(s, \chi)
$$

\footnotetext{
* The author was partially supported by the grant no. 2013/11/B/ST1/02799 from the National Science Centre.
} 
and

$$
A(\mathfrak{f})=(2 \pi)^{-r_{2}} \sqrt{|\Delta| N \mathfrak{f}} .
$$

Let $\Lambda(\mathfrak{a})$ be the generalized Mangoldt function. Fix $X \bmod \mathfrak{f} \in H$. We define,

$$
\Psi(x, X)=\sum_{\substack{x \leq N \mathfrak{a} \leq 2 x \\ \mathfrak{a} \in X}} \Lambda(\mathfrak{a})=\sum_{\substack{x \leq N \mathfrak{p}^{m} \leq 2 x \\ \mathfrak{p}^{m} \in X}} \log N \mathfrak{p},
$$

where $\mathfrak{p}$ runs through prime ideals of $\mathcal{O}_{K}$. The aim of this paper is to proof the following theorem.

Theorem 1. Let $K, \Delta, \mathfrak{f}, \zeta(s, \chi)$ denote respectively any algebraic number field of degree $[K: \mathbb{Q}]=2 r_{2}$, the discriminant of $K$, any ideal in $K$ and any HeckeLandau zeta function with a character $\chi$ modulo f. Fix $0<\varepsilon<1$. If $|\Delta| \geq 9$ and there is no zero in the region

$$
\sigma \geq 1-0.0795\left(\log |\Delta|+0.7761 \log \left((|t|+1)^{2 r_{2}}(N \mathfrak{f})^{1-E_{0}}\right)\right)^{-1},
$$

then

$$
\Psi(x, X) \geq \frac{x(1-\varepsilon)}{h_{\mathfrak{f}}^{*}(K)},
$$

for

$$
\log x \geq\left(23.148 \sqrt{r_{2}}\left(1+\left(2 \log \left(\frac{c_{1} \sqrt{r_{2}}}{0.117 \varepsilon}\right)\right)^{\frac{1}{2}}+\frac{2}{3} \log \left(\frac{c_{1} \sqrt{r_{2}}}{0.117 \varepsilon}\right)\right)\right)^{2} .
$$

where

$$
c_{1}=\left(36997.123|\Delta|^{\frac{1.933}{r_{2}}}+19064.499|\Delta|^{\frac{1.289}{r_{2}}}(N \mathfrak{f})^{\frac{1}{r_{2}}} h_{\mathfrak{f}}^{*}(K)\right) r_{2}^{2} \log (|\Delta| N \mathfrak{f}) .
$$

Remark 1. For real $\chi(\bmod \mathfrak{f})$ may exist in (11) one zero of $\zeta(s, \chi)$, which may be real and simple. However, we can check numerically that a Hecke-Landau $\zeta$ function has a simple real zero in (10) using scripts for computing zeros zeta functions associated to characters of finite order [12.

Explicit results of this kind are useful for estimating of computational complexity of algorithms which generates special primes. Such primes can be used in computational number theory and cryptography. In order to calculate exactly the time of the algorithms one need an explicit bound for the number of desired primes from the interval $[x, 2 x], x \geq x_{0}$, where $x_{0}$ is computed explicitly. We give an example of such an algorithm. For this reason we recall the definition [6].

Definition 1. Let $p, q$ be a pair of primes and $\Delta<0$. The primes $p, q$ are defined to be CM-primes with respect to $\Delta$ if there exist integers $f$ and $t$ such that

$$
|t| \leq 2 \sqrt{p}, \quad q \mid p+1-t, \quad 4 p-t^{2}=\Delta f^{2} .
$$


If CM-primes $p$ and $q$ with respect to $\Delta$ and integers $f, t$ are given, then an ordinary elliptic curve $E$ over $\mathbb{F}_{p}$ of cardinality $p+1-t$ can be constructed using complex multiplication method [1, 3]. Let $E\left(\mathbb{F}_{p}\right)$ be the group of points on $E$ over $\mathbb{F}_{p}$, and let $\left|E\left(\mathbb{F}_{p}\right)\right|$ be the order of $E\left(\mathbb{F}_{p}\right)$. The group $E\left(\mathbb{F}_{p}\right)$ can be used to implement public key cryptographic systems, based on intractability of the discrete logarithm problem (DLP). To make the DLP in $E\left(\mathbb{F}_{p}\right)$ intractable, it is essential to generate a large prime $p$, and a curve $E$ defined over $\mathbb{F}_{p}$, such that $\left|E\left(\mathbb{F}_{p}\right)\right|$ has a large prime factor $q$. In [6] an algorithmic method for constructing a pair $(E, p)$ such that $\left|E\left(\mathbb{F}_{p}\right)\right|$ has a large prime factor $q$ is given. Fix $K$ an imaginary quadratic number field, and positive integers $m, n,(n, m)=1$. Then the algorithm generates $\alpha \in \mathcal{O}_{K}$ such that $q=N_{K / \mathbb{Q}}(\alpha) \equiv m(\bmod n)$ is a prime, and $x \leq q \leq 2 x$ for sufficiently large $x \geq x_{0}$. Given $\alpha, q$ a prime $p$, $x<p<x^{\frac{5}{2-5 \varepsilon}}$, is constructed, where $0<\varepsilon<\frac{2}{5}$. For more algorithms of this kind we refer the reader to [7, [8].

Let $x \in \mathbb{R}$, and let $W(x)$ be the Lambert $W$ function such that $W(x) e^{W(x)}=$ $x$. If $-e^{-1} \leq x \leq 0$, then there are two possible real values of $W(x)$. We denote the branch satisfying $-1<W(x)$ by $W_{0}(x)$ and the branch satisfying $W(x) \leq-1$ by $W_{-1}(x)$. Fix $X(\bmod \mathfrak{f}) \in H$. We define

$$
\psi(x, X):=\sum_{\substack{N \mathfrak{p}^{m}<x \\ \mathfrak{p}^{m} \in X}} \log N \mathfrak{p},
$$

where $\mathfrak{p}$ runs through prime ideals of $\mathcal{O}_{K}$. Theorem 1 follows from Theorem 2

Theorem 2. Let $K, \Delta, \mathfrak{f}, \zeta(s, \chi)$ denote respectively any algebraic number field of degree $[K: \mathbb{Q}]=2 r_{2}$, the discriminant of $K$, any ideal in $K$ and any HeckeLandau zeta function with a character $\chi$ modulo $\mathfrak{f}$. Let $A_{0}=0.7761$. If $|\Delta| \geq 9$ and there is no zero in the region

$$
\sigma \geq 1-0.0795\left(\log |\Delta|+A_{0} \log \left((|t|+1)^{2 r_{2}}(N \mathfrak{f})^{1-E_{0}(\chi)}\right)\right)^{-1},
$$

then

$$
\psi(x, X) \geq \frac{x}{h_{\mathfrak{f}}^{*}(K)}-\frac{c_{2} x}{h_{\mathfrak{f}}^{*}(K)}(\log x)^{\frac{1}{2}} e^{-0.0432 r_{2}^{-1 / 2} \sqrt{\log x}},
$$

and

$$
\psi(x, X) \leq \frac{x}{h_{\mathfrak{f}}^{*}(K)}+\frac{c_{3} x}{h_{\mathfrak{f}}^{*}(K)}(\log x)^{\frac{1}{2}} e^{-0.0459 r_{2}^{-1 / 2} \sqrt{\log x}}
$$

for $x \geq \exp \left(116 r_{2} \log \left(2|\Delta|^{\frac{1}{A_{0} r_{2}}}(N \mathfrak{f})^{\frac{1}{r_{2}}}\right)\right)$, where

$$
\begin{aligned}
& c_{2}=\left(10756.659|\Delta|^{\frac{3}{2 A_{0} r_{2}}}+5541.374|\Delta|^{\frac{1}{A_{0} r_{2}}}(N \mathfrak{f})^{\frac{1}{r_{2}}} h_{\mathfrak{f}}^{*}(K)\right) r_{2}^{2} \log (|\Delta| N \mathfrak{f}), \\
& c_{3}=\left(14665.542|\Delta|^{\frac{3}{2 A_{0} r_{2}}}+7555.065|\Delta|^{\frac{1}{A_{0} r_{2}}}(N \mathfrak{f})^{\frac{1}{r_{2}}} h_{\mathfrak{f}}^{*}(K)\right) r_{2}^{2} \log (|\Delta| N \mathfrak{f}) .
\end{aligned}
$$

Proof. See Section 2 . 
We are now in a position to prove Theorem 1

Proof. By Theorem 2 we have

$$
\psi(2 x, X)-\psi(x, X) \geq \frac{x}{h_{\mathfrak{f}}^{*}(K)}-\frac{c_{1} x}{h_{\mathfrak{f}}^{*}(K)}(\log x)^{\frac{1}{2}} e^{-0.0432 r_{2}^{-1 / 2} \sqrt{\log x}},
$$

where

$$
c_{1}=\left(2 c_{2}\left(1+\frac{\log 2}{\log x}\right)^{\frac{1}{2}}+c_{3}\right) \leq 2.077 c_{2}+c_{3}
$$

for $x \geq \exp \left(116 r_{2} \log \left(2|\Delta|^{\frac{1}{A_{0} r_{2}}}(N \mathfrak{f})^{\frac{1}{r_{2}}}\right)\right)$. Fix $0<\varepsilon<1$. If

$$
c_{1}(\log x)^{\frac{1}{2}} e^{-0.0432 r_{2}^{-1 / 2} \sqrt{\log x}} \leq \varepsilon
$$

then

$$
0.0432 r_{2}^{-\frac{1}{2}}(\log x)^{\frac{1}{2}} \geq-W_{-1}\left(\frac{-0.0432 \varepsilon}{c_{1} \sqrt{r_{2}}}\right)
$$

By [2, Theorem 1]

$$
\log x \geq\left(23.148 \sqrt{r_{2}}\left(1+\left(2 \log \left(\frac{c_{1} \sqrt{r_{2}}}{0.117 \varepsilon}\right)\right)^{\frac{1}{2}}+\frac{2}{3} \log \left(\frac{c_{1} \sqrt{r_{2}}}{0.117 \varepsilon}\right)\right)^{2} .\right.
$$

This finishes the proof.

\section{The proof of Theorem 2}

The proof of Theorem 2 rests on the following lemmas and theorems.

Theorem 3. Let $K, \mathfrak{f}, \zeta(s, \chi)$ denote respectively any algebraic number field of degree $n \geq 2$, any ideal in $K$ and any Hecke-Landau zeta function with a character $\chi$ modulo $\mathfrak{f}$. Let futher

$$
L(t)=\log |\Delta|+A_{0} \log \left((|t|+1)^{n}(N \mathfrak{f})^{1-E_{0}}\right) \geq 2.097 .
$$

Then in the case of the complex $\chi$ in the region

$$
\sigma \geq 1-\frac{A_{1}}{L(t)} \geq 1-0.037911=0.962088=A_{2}
$$

there is no zero of $\zeta(s, \chi)$, where $A_{0}=0.7761, A_{1}=0.0795$. For real $\chi(\bmod \mathfrak{f})$ they may exist in (4) one zero of $\zeta(s, \chi)$, which may be real and simple.

Proof. See [5, Th. 2]. 
Lemma 1. Let $s=\sigma+i t, 0<\eta \leq \frac{1}{4}, A_{3}=75.472, A_{4}=0.010$ and $|\Delta| \geq 9$. Assume that there is no exceptional zero in the region (4) Then in the strip $1-\frac{A_{1}}{6 L(t)} \leq \sigma \leq 3$ we have.

$$
\left|\frac{\zeta^{\prime}}{\zeta}\left(s, \chi_{0}\right)+\frac{1}{s-1}\right| \leq \phi_{0}\left(t, r_{2}, \eta, \Delta, \mathfrak{f}\right)
$$

where

$$
\begin{gathered}
\phi_{0}\left(t, r_{2}, \eta, \Delta, \mathfrak{f}\right)=32 \log \left(L(t)(|t|+4)(|t|+2)^{r_{2}(1+\eta)}\left(1+A_{3} L(t)\right)^{2 r_{2}}\right) \\
+32 \log \left(A_{3}(|\Delta| N \mathfrak{f})^{\frac{1+\eta}{2}} \zeta(1+\eta)^{2 r_{2}}\right)+8 A_{3} r_{2} L(t)+\frac{A_{4} r_{2}}{L(t)}
\end{gathered}
$$

and

$$
\left|\frac{\zeta^{\prime}}{\zeta}(s, \chi)\right| \leq \phi\left(t, r_{2}, \eta, \Delta, \mathfrak{f}\right)
$$

where

$$
\begin{aligned}
& \phi\left(t, r_{2}, \eta, \Delta, \mathfrak{f}\right)=32 \log \left(\left(1+A_{3} L(t)\right)^{2 r_{2}}(|t|+2)^{r_{2}(1+2 \eta)}\right) \\
& \quad+32 \log \left(1.4\left(1+\varepsilon_{\chi}\right) A(\mathfrak{f})^{1+2 \eta} \zeta(1+\eta)^{2 r_{2}}\right)+4 A_{3} r_{2} L(t)+\frac{A_{4} r_{2}}{L(t)}
\end{aligned}
$$

for any character $\chi \neq \chi_{0}$ modulo $\mathfrak{f}$, where $\varepsilon_{\chi}=0$ or 1 to accordingly whether $\chi$ is primitive or not.

Proof. See Section 3

Lemma 2. Let $\phi_{0}$, $\phi$ be functions defined in Lemma 1, Let $T \geq 1, w \geq 1$, $|\Delta| \geq 9, c_{4}=\frac{1}{\sqrt{2 w r_{2}}}$ and

$$
c_{0}=c_{0}\left(\Delta, \mathfrak{f}, r_{2}, E_{0}\right)=|\Delta|^{-\frac{1}{2 A_{0} r_{2}}}(N \mathfrak{f})^{-\frac{1-E_{0}}{2 r_{2}}}
$$

If

$$
T+1=c_{0} \exp \left(c_{4} \sqrt{\log x}\right)
$$

then

$$
\begin{aligned}
\phi\left(T, r_{2}, \eta, \Delta, \mathfrak{f}\right) & \leq 230.911 r_{2}^{\frac{3}{2}} \log (|\Delta| N \mathfrak{f})(\log x)^{\frac{1}{2}} \\
\phi_{0}\left(T, r_{2}, \eta, \Delta, \mathfrak{f}\right) & \leq 412.531 r_{2}^{\frac{3}{2}} \log (|\Delta| N \mathfrak{f})(\log x)^{\frac{1}{2}}
\end{aligned}
$$

for $x \geq \exp \left(\left(c_{4}^{-1} \log \left(2 c_{0}^{-1}\right)\right)^{2}\right)$. 
Proof. Since $T+1 \geq 2, \log x \geq 2 r_{2} w\left(\log \left(2 c_{0}^{-1}\right)\right)^{2} \geq 2\left(\log \left(2 \cdot 9^{\frac{1}{2 A_{0}}}\right)\right)^{2}=8.892$, and hence $x>e^{8.892}$. By (3), (8) we obtain

$$
L(T)=\frac{A_{0} \sqrt{2 r_{2}}}{\sqrt{w}}(\log x)^{\frac{1}{2}}, \quad L(T) \geq 2.097 .
$$

Let $|\Delta| \geq 9, x>e^{8.892}$ and $\eta=\frac{1}{4}$. We have $\zeta\left(\frac{5}{4}\right) \leq 4.596$,

$$
\begin{aligned}
& 32 \log \left(1+A_{3} L(T)\right)^{2 r_{2}} \leq 32 r_{2} \log \log x+64 r_{2} \log \left(A_{0} \sqrt{2 r_{2}}\left(A_{3}+\frac{1}{2.097}\right)\right) \\
& \leq 111.419 r_{2}^{\frac{3}{2}} \log \log x \\
& 32 r_{2}(1+2 \eta) \log (T+2) \leq \frac{32(1+2 \eta) \sqrt{r_{2}}}{\sqrt{2}}(\log x)^{\frac{1}{2}} \leq 37.336 r_{2}^{\frac{1}{2}}(\log x)^{\frac{1}{2}}, \\
& 4 A_{3} r_{2} L(T) \leq 4 \sqrt{2} A_{0} A_{3} r_{2}^{\frac{3}{2}}(\log x)^{\frac{1}{2}} \leq 331.334 r_{2}^{\frac{3}{2}}(\log x)^{\frac{1}{2}}, \\
& 32 \log \left(1.4\left(1+\varepsilon_{\chi}\right) A(\mathfrak{f})^{1+2 \eta} \zeta(1+\eta)^{2 r_{2}}\right)+\frac{A_{4} r_{2}}{L(T)} \leq 32 \log \left(2.8 \zeta(1+\eta)^{2 r_{2}}\right) \\
&+16(1+2 \eta) \log (|\Delta| N \mathfrak{f})+\frac{A_{4} r_{2}}{2.097} \leq 73.419 r_{2} \log (|\Delta| N \mathfrak{f}) .
\end{aligned}
$$

By the above and (6) we obtain

$$
\phi\left(T, r_{2}, \eta, \Delta, \mathfrak{f}\right) \leq 230.911 r_{2}^{\frac{3}{2}} \log (|\Delta| N \mathfrak{f})(\log x)^{\frac{1}{2}},
$$

Similarly,

$$
\begin{aligned}
32 \log L(T) \leq & 16 \log \log x+32 \log \left(\sqrt{2 r_{2}} A_{0}\right) \leq 24.686 r_{2}^{\frac{1}{2}} \log \log x, \\
& 32 \log (T+4)^{r_{2}(1+\eta)+1} \leq 56.3 r_{2}(\log x)^{\frac{1}{2}}
\end{aligned}
$$

and

$$
32 \log \left(A_{3}(|\Delta| N \mathfrak{f})^{\frac{1+\eta}{2}} \zeta(1+\eta)^{2 r_{2}}\right)+\frac{A_{4} r_{2}}{L(t)} \leq 84.361 r_{2} \log (|\Delta| N \mathfrak{f}) .
$$

By the above and (5) we obtain

$$
\phi_{0}\left(T, r_{2}, \eta, \Delta, \mathfrak{f}\right) \leq 412.531 r_{2}^{\frac{3}{2}} \log (|\Delta| N \mathfrak{f})(\log x)^{\frac{1}{2}} .
$$

This finishes the proof. 
Lemma 3. Let $T \geq 1, w \geq 1,|\Delta| \geq 9$, and let $k \geq 1$. Let $c_{0}$ be the constant appearing in (7). If

$$
T+1=c_{0} \exp \left(\sqrt{\frac{\log x}{2 w r_{2}}}\right)
$$

then

$$
\begin{gathered}
\frac{1}{T^{k}} \leq 2^{k} c_{0}^{-k} e^{-k c_{4} \sqrt{\log x}} \quad \text { for } \quad \log x \geq\left(c_{4}^{-1} \log \left(2 c_{0}^{-1}\right)\right)^{2}, \\
\log (e(T+k)) \leq c_{4} \sqrt{\log x}+\log \left(e\left(\frac{k+1}{2}\right)\right) .
\end{gathered}
$$

Proof. By (10) we have

$$
\frac{1}{T^{k}}=\exp \left(-k \log \left(c_{0} e^{c_{4} \sqrt{\log x}}\left(1-\left(c_{0} e^{c_{4} \log x}\right)^{-1}\right)\right)\right) \leq \exp \left(-k \log \left(\frac{1}{2} c_{0} e^{c_{4} \sqrt{\log x}}\right)\right),
$$

for $\log x \geq\left(c_{4}^{-1} \log \left(2 c_{0}^{-1}\right)\right)^{2}$. The proof of (12) is left to the reader. This finishes the proof.

Lemma 4. For $T \geq 1$ we have

$$
\int_{T}^{\infty} t^{-2} d t \leq T^{-1}, \quad \int_{T}^{\infty} t^{-2} \log (t+4) d t \leq T^{-1} \log (e(T+4))
$$

Proof. The proof is left to the reader.

Lemma 5. Let $L(t)$ be the function which occur in (3). For $T \geq 1$ we have

$$
\int_{T}^{\infty} t^{-2} L(t) d t \leq c_{5} T^{-1} \log (e(T+4))
$$

where $c_{5}=1.09 r_{2} \log \left(|\Delta|(N \mathfrak{f})^{A_{0}\left(1-E_{0}\right)}\right)$.

Proof. We have

$$
\int_{T}^{\infty} t^{-2} L(t) d t \leq 2 r_{2} A_{0} \int_{T}^{\infty} t^{-2} \log (t+4) d t+\log \left(|\Delta|(N \mathfrak{f})^{A_{0}\left(1-E_{0}\right)}\right) \int_{T}^{\infty} t^{-2} d t .
$$

The Lemma 5 follows from Lemma 4 This finishes the proof.

Lemma 6. Let $L(t)$ be the function which occur in (3). For $T \geq 1$ we have

$$
\int_{T}^{\infty} t^{-2} \log \left(1+A_{3} L(t)\right)^{2 r_{2}} d t \leq c_{6} T^{-1} \log (e(T+4)),
$$

where $c_{6}=11.605 r_{2}^{2} \log \left(|\Delta|(N \mathfrak{f})^{A_{0}\left(1-E_{0}\right)}\right)$, and $A_{3}$ is the constant appearing in Lemma 1 . 
Proof. By (3) we have

$$
\int_{T}^{\infty} t^{-2} \log \left(1+A_{3} L(t)\right)^{2 r_{2}} d t \leq 2 r_{2} c_{7} \int_{T}^{\infty} t^{-2} d t+2 r_{2} \int_{T}^{\infty} t^{-2} L(t) d t
$$

where $c_{7}=\log \left(A_{3}\left(1+\frac{1}{2.097 A_{3}}\right)\right)$. The Lemma 6 follows from Lemma 4 and Lemma 5. This finishes the proof.

Lemma 7. Let $\phi_{0}$ be the function which occur in (5). For $T \geq 1$ we have

$$
\int_{T}^{\infty} \phi_{0}\left(t, r_{2}, \eta, \Delta, \mathfrak{f}\right) t^{-2} d t \leq c_{8} T^{-1} \log (e(T+4)),
$$

where $c_{8}=1138.428 r_{2}^{2} \log \left(|\Delta|(N \mathfrak{f})^{\frac{5}{8}}\right)$.

Proof. By (3) and Lemma 1 with $\eta=\frac{1}{4}$ we have

$$
\begin{aligned}
& \int_{T}^{\infty} \phi_{0}\left(t, r_{2}, \eta, \Delta, \mathfrak{f}\right) t^{-2} d t \leq\left(40 r_{2}+32\right) \int_{T}^{\infty} t^{-2} \log (t+4) d t \\
& \quad+\left(32 \log \left(A_{3}(|\Delta| N \mathfrak{f})^{\frac{5}{8}}\right)+64 r_{2} \log \zeta\left(\frac{5}{4}\right)+\frac{A_{4} r_{2}}{2.097}\right) \int_{T}^{\infty} t^{-2} d t \\
& \quad+\left(32+8 A_{3} r_{2}\right) \int_{T}^{\infty} t^{-2} L(t) d t+32 \int_{T}^{\infty} t^{-2} \log \left(1+A_{3} L(t)\right)^{2 r_{2}} d t
\end{aligned}
$$

The Lemma 7 follows from Lemmas 4, 5 and 6. This finishes the proof.

Lemma 8. Let $\phi$ be the function which occur in (6). For $T \geq 1$ we have

$$
\int_{T}^{\infty} \phi\left(t, r_{2}, \eta, \Delta, \mathfrak{f}\right) t^{-2} d t \leq c_{9} T^{-1} \log (e(T+4)),
$$

where $c_{9}=821.212 r_{2}^{2} \log (|\Delta| N \mathfrak{f})$.

Proof. By (3) and Lemma 1 with $\eta=\frac{1}{4}$ we have

$$
\begin{aligned}
& \int_{T}^{\infty} \phi\left(t, r_{2}, \eta, \Delta, \mathfrak{f}\right) t^{-2} d t \leq\left(32 \log \left(2.8 A(\mathfrak{f})^{\frac{3}{2}} \zeta\left(\frac{5}{4}\right)^{2 r_{2}}\right)+\frac{A_{4} r_{2}}{2.097}\right) \int_{T}^{\infty} t^{-2} d t \\
& \quad+32 \int_{T}^{\infty} t^{-2} \log \left(1+A_{3} L(t)\right)^{2 r_{2}} d t+16 r_{2} \int_{T}^{\infty} t^{-2} \log (t+4) d t \\
& \quad+4 A_{3} r_{2} \int_{T}^{\infty} L(t) t^{-2} d t .
\end{aligned}
$$

The Lemma 8 follows from Lemmas 4 , 5 and 6 . This finishes the proof. 
We are now in a position to prove Theorem 2

Proof. Fix $T \geq 1$, and let $c=1+\frac{1}{\log x}$. Fix $X(\bmod \mathfrak{f})$. We define

$$
\psi_{1}(x, X):=\int_{0}^{x} \psi(t, X) d t
$$

and

$$
\gamma(n)=\sum_{\substack{N \mathfrak{p}^{m}=n \\ \mathfrak{p}^{m} \in X}} \log N \mathfrak{p}
$$

Hence,

$$
\psi(x, X)=\sum_{n \leq x} \gamma(n)
$$

By partial summation we obtain

$$
\sum_{n \leq x}(x-n) \gamma(n)=\int_{0}^{x} \psi(t, X) d t
$$

Now, we write

$$
f(s, \chi)=\frac{x^{s-1}}{s(s+1)}\left[-\frac{\zeta^{\prime}}{\zeta}(s, \chi)\right] .
$$

By Theorem B [9, see p. 31] and the orthogonality properties of $\chi(\bmod \mathfrak{f})$ we deduce the formula

$$
\sum_{n \leq x}(x-n) \gamma(n)=\frac{x^{2}}{2 \pi i h_{\mathfrak{f}}^{*}(K)} \sum_{\chi} \bar{\chi}(X) \int_{c-i \infty}^{c+i \infty} f(s, \chi) d s
$$

where $c>1$. Let $A_{1}$ be the constat appearing in (4), and let $B=\frac{A_{1}}{6}=0.0133$. We define the contour $\mathcal{C}$ consisting of the following parts:

$$
\begin{aligned}
& \mathcal{C}_{1}: s=c+i t, \text { where }-T \leq t \leq T, \\
& \mathcal{C}_{2}: s=\sigma+i T, \text { where } 1-\frac{B}{L(T)} \leq \sigma \leq c, \\
& \mathcal{C}_{3}: s=1-\frac{B}{L(t)}+i t, \text { where }-T \leq t \leq T .
\end{aligned}
$$

and of $\mathcal{C}_{2}^{\prime}$ situated symmetrically to $\mathcal{C}_{2}$. If $\chi=\chi_{0}$, them $\frac{\zeta^{\prime}}{\zeta}(s, \chi)$ has a first order pole of residue -1 at $s=1$. From the Cauchy formula we get

$$
\frac{1}{2 \pi i} \int_{\mathcal{C}_{1}} f(s, \chi) d s=\frac{\delta(\chi)}{2}-\frac{1}{2 \pi i} \int_{\mathcal{C}_{2}+\mathcal{C}_{3}+\mathcal{C}_{2}^{\prime}} f(s, \chi) d s
$$


where

$$
\delta(\chi)= \begin{cases}1 & \text { if } \chi=\chi_{0} \\ 0 & \text { if } \chi \neq \chi_{0}\end{cases}
$$

From (13), (14) and (16) we obtain

$$
\left|\psi_{1}(x, X)-\frac{x^{2}}{2 h_{\mathfrak{f}}^{*}(K)}\right| \leq \frac{x^{2}\left(I_{1}+I_{2}+I_{3}\right)}{h_{\mathfrak{f}}^{*}(K)}+\frac{x^{2}\left(J_{1}+J_{2}+J_{3}\right)}{h_{\mathfrak{f}}^{*}(K)},
$$

where

$$
\begin{aligned}
I_{1}+I_{2}+I_{3} & =\left|\frac{1}{2 \pi i} \int_{c-i \infty}^{c-i T} f\left(s, \chi_{0}\right)\right|+\left|\frac{1}{2 \pi i} \int_{\mathcal{C}_{2}+\mathcal{C}_{3}+\mathcal{C}_{2}^{\prime}} f\left(s, \chi_{0}\right) d s\right|+\left|\frac{1}{2 \pi i} \int_{c+i T}^{c+i \infty} f\left(s, \chi_{0}\right) d s\right|, \\
J_{1}+J_{2}+J_{3} & =\left|\sum_{\chi \neq \chi_{0}} \bar{\chi}(X) \frac{1}{2 \pi i} \int_{c-i \infty}^{c-i T} f(s, \chi) d s\right|+\left|\sum_{\chi \neq \chi_{0}} \bar{\chi}(X) \frac{1}{2 \pi i} \int_{\mathcal{C}_{2}+\mathcal{C}_{3}+\mathcal{C}_{2}^{\prime}} f(s, \chi) d s\right| \\
& +\left|\sum_{\chi \neq \chi_{0}} \bar{\chi}(X) \frac{1}{2 \pi i} \int_{c+i T}^{c+i \infty} f(s, \chi) d s\right| .
\end{aligned}
$$

We define

$$
h_{0}\left(s, \chi_{0}\right)=\left[-\frac{\zeta^{\prime}}{\zeta}\left(s, \chi_{0}\right)-\frac{1}{s-1}\right] \frac{x^{s-1}}{s(s+1)}, \quad h_{1}(s)=\frac{x^{s-1}}{s(s+1)(s-1)} .
$$

We estimate the above integrals. Let $T \geq 1, x \geq e^{8.892}, 1<c \leq 1+\frac{1}{\log x} \leq 1.12$. We write We need to consider the following cases:

1. Bound over $\mathcal{C}_{2}$ and $\mathcal{C}_{2}^{\prime}$, case $\chi=\chi_{0}$. From Lemmas 2 and 3 we obtain

$$
\begin{aligned}
& \left|\frac{1}{2 \pi i} \int_{\mathcal{C}_{2}} f\left(\sigma+i T, \chi_{0}\right) d \sigma\right| \leq \frac{e}{2 \pi T^{2} \log x} \phi_{0}\left(T, r_{2}, \eta, \Delta, \mathfrak{f}\right)+\frac{e}{2 \pi T^{3} \log x} \\
& \quad \leq c_{0}^{-3} r_{2}^{\frac{3}{2}} \log (|\Delta| N \mathfrak{f})(\log x)^{-\frac{1}{2}}\left(\frac{412.531 c_{0} e}{\pi}+\frac{4 c_{0} e}{(\log x)^{\frac{1}{2}}}\right) e^{-2 c_{4} \sqrt{\log x}} \\
& \quad \leq c_{10}(\log x)^{-\frac{1}{2}} e^{-2 c_{4} \sqrt{\log x}}
\end{aligned}
$$

where $c_{10}=360.992|\Delta|^{\frac{3}{2 A_{0} r_{2}}} r_{2}^{\frac{3}{2}} \log (|\Delta| N \mathfrak{f})$. The same bound holds with $\int_{\mathcal{C}_{2}^{\prime}}$ in place of $\int_{\mathcal{C}_{2}}$.

2. Bound over $\mathcal{C}_{3}$, case $\chi=\chi_{0}$. Lemmas 2 and 3 shows that

$$
\begin{aligned}
& \left|\frac{1}{2 \pi i} \int_{\mathcal{C}_{3}} h_{0}\left(1-\frac{B}{L(T)}+i t, \chi_{0}\right) d t\right| \leq \frac{1}{\pi} x^{-\frac{B}{L(T)}} \phi_{0}\left(T, r_{2}, \eta, \Delta, \mathfrak{f}\right) \int_{0}^{T} \frac{d t}{\left(1-\frac{B}{L(T)}\right)^{2}+t^{2}} \\
& \quad \leq \frac{1}{\pi} 2.01 e^{-c_{18} \sqrt{\log x}} \phi_{0}\left(T, r_{2}, \eta, \Delta, \mathfrak{f}\right) \leq 263.939 r_{2}^{\frac{3}{2}} \log (|\Delta| N \mathfrak{f})(\log x)^{\frac{1}{2}} e^{-c_{18} \sqrt{\log x}}
\end{aligned}
$$


where $c_{18}=\frac{B \sqrt{w}}{A_{0} \sqrt{2 r_{2}}}$. Indeed, $1-\frac{B}{L(T)} \geq 1-\frac{0.0133}{2.097}=0.993$, and

$$
\begin{aligned}
& \int_{0}^{T} \frac{d t}{\left(1-\frac{B}{L(T)}\right)^{2}+t^{2}}=\int_{0}^{1} \frac{d t}{\left(1-\frac{B}{L(T)}\right)^{2}+t^{2}}+\int_{1}^{T} \frac{d t}{\left(1-\frac{B}{L(T)}\right)^{2}+t^{2}} \\
& \quad \leq \int_{0}^{1} \frac{d t}{(0.993)^{2}}+\int_{1}^{T} \frac{d t}{t^{2}} \leq \frac{1}{(0.993)^{2}}+1=2.01 .
\end{aligned}
$$

Moreover,

$$
\begin{aligned}
& \left|\frac{1}{2 \pi i} \int_{\mathcal{C}_{3}} h_{1}\left(1-\frac{B}{L(t)}+i t\right) d s\right| \leq \frac{1}{\pi} x^{-\frac{B}{L(T)}} \int_{0}^{T} \frac{d t}{\left|1-\frac{B}{L(T)}+t\right|\left|2-\frac{B}{L(T)}+t\right|\left|-\frac{B}{L(T)}+t\right|} \\
& \quad \leq \frac{c_{11}}{\pi} e^{-c_{18} \sqrt{\log x}} .
\end{aligned}
$$

where $c_{11}=\frac{1}{0.993|0.993-1|(0.993+1)}+1 \leq 73.185$. By the above and (18),

$$
\left|\frac{1}{2 \pi i} \int_{\mathcal{C}_{3}} f\left(1-\frac{B}{L(T)}+i t, \chi_{0}\right) d t\right| \leq c_{12}(\log x)^{\frac{1}{2}} e^{-c_{18} \sqrt{\log x}},
$$

where $c_{12}=267.495 r_{2}^{\frac{3}{2}} \log (|\Delta| N \mathfrak{f})$. Hence,

$$
\begin{aligned}
I_{2} & \leq|\Delta|^{\frac{3}{2 A_{0} r_{2}}} r_{2}^{\frac{3}{2}} \log (|\Delta| N \mathfrak{f})(\log x)^{\frac{1}{2}} e^{-c_{18} \sqrt{\log x}} \\
& \cdot\left(267.495+\frac{2 \cdot 360.992}{\log x} e^{-\left(2 c_{4}-c_{18}\right) \sqrt{\log x}}\right) \leq c_{13}(\log x)^{\frac{1}{2}} e^{-c_{18} \sqrt{\log x}},
\end{aligned}
$$

if $w<\frac{2 A_{0}}{B}=117.14$, where $c_{13}=348.69|\Delta|^{\frac{3}{2 A_{0} r_{2}}} r_{2}^{\frac{3}{2}} \log (|\Delta| N \mathfrak{f})$.

3. Bound over $\mathcal{C}_{2}$ and $\mathcal{C}_{2}^{\prime}$, case $\chi \neq \chi_{0}$. From Lemmas 2 and 3 we obtain

$$
\left|\frac{1}{2 \pi i} \int_{\mathcal{C}_{2}} f\left(\sigma+i T, \chi_{0}\right) d s\right| \leq c_{14}(\log x)^{-\frac{1}{2}} e^{-2 c_{4} \sqrt{\log x}},
$$

where $c_{14}=399.594|\Delta|^{\frac{1}{A_{0} r_{2}}}(N \mathfrak{f})^{\frac{1}{r_{2}}} r_{2}^{\frac{3}{2}} \log (|\Delta| N \mathfrak{f})$. The same bound holds with $\int_{\mathcal{C}_{2}^{\prime}}$ in place of $\int_{\mathcal{C}_{2}}$.

4. Bound over $\mathcal{C}_{3}$, case $\chi \neq \chi_{0}$. Lemmas 2 and 3 shows that

$$
\begin{aligned}
& \left|\frac{1}{2 \pi i} \int_{\mathcal{C}_{3}} f\left(1-\frac{B}{L(T)}+i t, \chi_{0}\right) d s\right| \leq \frac{1}{\pi} x^{-\frac{B}{L(T)}} \phi\left(T, r_{2}, \eta, \Delta, \mathfrak{f}\right) \int_{0}^{T} \frac{d t}{\left(1-\frac{B}{L(T)}\right)^{2}+t^{2}} \\
& \quad \leq \frac{1}{\pi} 2.01 e^{-c_{18} \sqrt{\log x}} \phi\left(T, r_{2}, \eta, \Delta, \mathfrak{f}\right) \leq c_{15}(\log x)^{\frac{1}{2}} e^{-c_{18} \sqrt{\log x}},
\end{aligned}
$$


where $c_{15}=147.738 r_{2}^{\frac{3}{2}} \log (|\Delta| N \mathfrak{f})$. Hence, by the above

$$
\begin{aligned}
J_{2} & \leq 2 c_{14}(\log x)^{\frac{1}{2}} e^{-2 c_{4} \sqrt{\log x}}+c_{15}(\log x)^{\frac{1}{2}} e^{-c_{18} \sqrt{\log x}} \\
& \leq c_{16}(\log x)^{\frac{1}{2}} e^{-c_{18} \sqrt{\log x}}
\end{aligned}
$$

if $w<\frac{2 A_{0}}{B}=117.14$, where $c_{16}=237.616|\Delta|^{\frac{1}{A_{0} r_{2}}}(N \mathfrak{f})^{\frac{1}{r_{2}}} r_{2}^{\frac{3}{2}} \log (|\Delta| N \mathfrak{f}) .5$. Bound for $\int_{c+i T}^{c+i \infty}$, case $\chi=\chi_{0}$. By (18) and Lemmas 3, 7] we obtain

$$
\begin{aligned}
& \left|\frac{1}{2 \pi i} \int_{c+i T}^{c+i \infty} f\left(s, \chi_{0}\right) d s\right| \leq\left|\frac{1}{2 \pi i} \int_{c+i T}^{c+i \infty} h_{0}\left(s, \chi_{0}\right) d s\right|+\left|\frac{1}{2 \pi i} \int_{c+i T}^{c+i \infty} h_{1}(s) d s\right| \\
& \quad \leq \frac{e}{2 \pi} \int_{T}^{\infty} \phi_{0}\left(t, r_{2}, \eta, \Delta, \mathfrak{f}\right) t^{-2} d t+\frac{e}{2 \pi} \int_{T}^{\infty} t^{-3} d t \leq \frac{e}{2 \pi} c_{8} \frac{\log (e(T+4))}{T} \\
& \quad+\frac{e}{4 \pi T^{2}} \leq \frac{e c_{8}}{\pi c_{0}^{2}}(\log x)^{\frac{1}{2}}\left(\frac{c_{0}}{\sqrt{2 w}}+\frac{1.917 c_{0}}{(\log x)^{\frac{1}{2}}}+\frac{c_{0}}{2 c_{8}(\log x)^{\frac{1}{2}}}\right) e^{-c_{4} \sqrt{\log x}} \\
& \leq c_{17}(\log x)^{\frac{1}{2}} e^{-c_{4} \sqrt{\log x}}
\end{aligned}
$$

for $\log x \geq\left(c_{4}^{-1} \log \left(2 c_{0}^{-1}\right)\right)^{2} \geq 8.892$, with $(\log x)^{\frac{1}{2}} \geq 2.98, c_{0} \leq 1, w=58$. where $c_{17}=724.845|\Delta|^{\frac{1}{A_{0} r_{2}}} r_{2}^{2} \log \left(|\Delta|(N \mathfrak{f})^{\frac{5}{8}}\right)$. The same bound holds with $\int_{c-i \infty}^{c-i T}$ in place of $\int_{c+i T}^{c+i \infty}$. Hence,

$$
I_{1}+I_{3} \leq 2 c_{17}(\log x)^{\frac{1}{2}} e^{-c_{4} \sqrt{\log x}} .
$$

6. Bound for $\int_{c+i T}^{c+i \infty}$, case $\chi \neq \chi_{0}$. Lemmas 3 and 8 shows that

$$
\begin{aligned}
& \left|\frac{1}{2 \pi i} \int_{c+i T}^{c+i \infty} f(s, \chi) d s\right| \leq \frac{e}{2 \pi} \int_{T}^{\infty} \phi\left(t, r_{2}, \eta, \Delta, \mathfrak{f}\right) t^{-2} d t \leq \frac{e}{2 \pi} c_{9} \frac{\log (e(T+4))}{T} \\
& \quad \leq \frac{e c_{9}}{\pi c_{0}}(\log x)^{\frac{1}{2}}\left(\frac{1}{\sqrt{2 w}}+\frac{1.917}{(\log x)^{\frac{1}{2}}}\right) e^{-c_{4} \sqrt{\log x}} \leq c_{19}(\log x)^{\frac{1}{2}} e^{-c_{4} \sqrt{\log x}} .
\end{aligned}
$$

where $c_{19}=522.77|\Delta|^{\frac{1}{2 A_{0} r_{2}}}(N \mathfrak{f})^{\frac{1}{2 r_{2}}} r_{2}^{2} \log (|\Delta| N \mathfrak{f})$, and $w=58$. The same bound holds with $\int_{c-i \infty}^{c-i T}$ in place of $\int_{c+i T}^{c+i \infty}$. Hence,

$$
J_{1}+J_{3} \leq 2 c_{19}(\log x)^{\frac{1}{2}} e^{-c_{4} \sqrt{\log x}} .
$$

By (19), (21) we have

$$
I_{1}+I_{2}+I_{3} \leq c_{20}(\log x)^{\frac{1}{2}} e^{-c_{18} \sqrt{\log x}},
$$

where $c_{20}=3585.536|\Delta|^{\frac{3}{2 A_{0} r_{2}}} r_{2}^{2} \log (|\Delta| N \mathfrak{f})$, for $1 \leq w<\frac{A_{0}}{B}=58.57$. From (20), (22) we obtain

$$
J_{1}+J_{2}+J_{3} \leq c_{21}(\log x)^{\frac{1}{2}} e^{-c_{18} \sqrt{\log x}},
$$


where $c_{21}=1847.116 h_{\mathfrak{f}}^{*}(K)|\Delta|^{\frac{1}{A_{0} r_{2}}}(N \mathfrak{f})^{\frac{1}{r_{2}}} r_{2}^{2} \log (|\Delta| N \mathfrak{f})$ for $1 \leq w<\frac{A_{0}}{B}=$ 58.57. Now, by (17), (23), (24) we obtain

$$
\left|\psi_{1}(x, X)-\frac{x^{2}}{2 h_{\mathfrak{f}}^{*}(K)}\right| \leq \frac{x^{2}}{h_{\mathfrak{f}}^{*}(K)} c_{22}(\log x)^{\frac{1}{2}} e^{-c_{18} \sqrt{\log x}}
$$

where $c_{22}=c_{20}+c_{21}$. Now, let $x>2$, and $h$ be a function of $x$ satisfying $0<h<\frac{1}{2} x$. Let $W(x)=c_{22}(\log x)^{\frac{1}{2}} e^{-c_{18} \sqrt{\log x}}$. Since $\psi(t, X)$ is an increasing function

$$
\begin{aligned}
\psi(x, X) & \geq \frac{1}{h} \int_{x-h}^{x} \psi(t, X) d t=\frac{\psi_{1}(x, X)-\psi_{1}(x-h, X)}{h} \\
\geq & \frac{x}{h_{\mathfrak{f}}^{*}(K)}-\frac{x^{2}}{h h_{\mathfrak{f}}^{*}(K)} W(x)-\frac{h}{2 h_{\mathfrak{f}}^{*}(K)}-\frac{x^{2}+h^{2}}{h h_{\mathfrak{f}}^{*}(K)} W(x-h) .
\end{aligned}
$$

Taking $h=x e^{-\frac{1}{2} c_{18} \sqrt{\log x}}$ and $x>\left(\frac{2 \log 2}{c_{18}}\right)^{2}$, we get

$$
\begin{aligned}
\psi(x, X) & \geq \frac{x}{h_{\mathfrak{f}}^{*}(K)}-\frac{x}{h_{\mathfrak{f}}^{*}(K)} c_{22}(\log x)^{\frac{1}{2}} e^{-\frac{1}{2} c_{18} \sqrt{\log x}}-\frac{1}{2 h_{\mathfrak{f}}^{*}(K)} x e^{-\frac{1}{2} c_{18} \sqrt{\log x}} \\
& -\frac{x}{h_{\mathfrak{f}}^{*}(K)} c_{22}(\log x)^{\frac{1}{2}} e^{-c_{18}\left(c_{23}-0.5\right) \sqrt{\log x}}+\frac{x}{h_{\mathfrak{f}}^{*}(K)} c_{22}(\log x)^{\frac{1}{2}} e^{-c_{18}\left(c_{23}+0.5\right) \sqrt{\log x}} \\
& \geq \frac{x}{h_{\mathfrak{f}}^{*}(K)}-\frac{x}{h_{\mathfrak{f}}^{*}(K)} c_{22}(\log x)^{\frac{1}{2}} e^{-0.47 c_{18} \sqrt{\log x}}\left(3+c_{24}\right) \\
& \geq \frac{x}{h_{\mathfrak{f}}^{*}(K)}-\frac{c_{2} x}{h_{\mathfrak{f}}^{*}(K)}(\log x)^{\frac{1}{2}} e^{-0.47 c_{18} \sqrt{\log x}}
\end{aligned}
$$

where $c_{23}=\left(1-\frac{\log 2}{\log x}\right)^{\frac{1}{2}}, 0.97 \leq c_{23} \leq 0.98, c_{24}=\frac{1}{2 c_{22}}(\log x)^{-\frac{1}{2}} \leq 0.0001$, and $c_{2}=c_{22}\left(3+c_{24}\right)$. On the other hand,

$$
\begin{aligned}
\psi(x, X) & \leq \frac{1}{h} \int_{x}^{x+h} \psi(t, X) d t=\frac{\psi_{1}(x+h, X)-\psi_{1}(x, X)}{h} \\
\leq & \frac{x}{h_{\mathfrak{f}}^{*}(K)}+\frac{h}{2 h_{\mathfrak{f}}^{*}(K)}+\frac{(x+h)^{2}}{h h_{\mathfrak{f}}^{*}(K)} W(x+h)+\frac{x^{2}}{h h_{\mathfrak{f}}^{*}(K)} W(x) \\
\leq & \frac{x}{h_{\mathfrak{f}}^{*}(K)}+\frac{x}{h_{\mathfrak{f}}^{*}(K)} c_{22}(\log x)^{\frac{1}{2}} e^{-\frac{1}{2} c_{18} \sqrt{\log x}}\left(c_{25}+5 c_{1}\right) \\
\leq & \frac{x}{h_{\mathfrak{f}}^{*}(K)}+\frac{c_{3} x}{h_{\mathfrak{f}}^{*}(K)}(\log x)^{\frac{1}{2}} e^{-\frac{1}{2} c_{18} \sqrt{\log x}}
\end{aligned}
$$

where $c_{25}=\frac{1}{2 c_{22} c_{26}}(\log x)^{-\frac{1}{2}} \leq 0.001, c_{26}=\left(1+\frac{\log \frac{3}{2}}{\log x}\right)^{\frac{1}{2}} \leq 1.013, c_{3}=c_{22}\left(c_{25}+\right.$ $5 c_{26}$ ). Putting $c_{18}=\frac{B \sqrt{58}}{A_{0} \sqrt{2 r_{2}}}=0.0919 \sqrt{r_{2}}$ we obtain the result. This finishes the proof. 


\section{Proof of Lemma 1}

The proof of Lemma 1 rest on the following lemmas.

Lemma 9. Let $[K: \mathbb{Q}]=2 r_{2}$ and $0<\eta \leq \frac{1}{4}$. In the region $-\eta \leq \sigma \leq 3$ we have the estimate

$$
|\zeta(\sigma+i t, \chi)| \leq 1.4^{r_{2}}\left(1+\varepsilon_{\chi}\right) A(\mathfrak{f})^{1+2 \eta} \zeta(1+\eta)^{2 r_{2}}(|t|+1)^{r_{2}(1+2 \eta)}
$$

for any character $\chi \neq \chi_{0}$ modulo $\mathfrak{f}$, where $\varepsilon_{\chi}=0$ or 1 to accordingly whether $\chi$ is primitive or not.

Proof. Consider

$$
g(s, \chi)=\frac{\zeta(s, \chi)}{\zeta(1-s, \bar{\chi})}
$$

where $\chi$ is a primitive character $\bmod \mathfrak{f}$. From the functional equation for $\zeta(s, \chi)$ it follows that

$$
g(s, \chi)=W(\chi) A(\mathfrak{f})^{1-2 s}\left(\frac{\Gamma(1-s)}{\Gamma(s)}\right)^{r_{2}}
$$

We estimate $g(s, \chi)$ on the line $s=-\eta+i t, 0 \leq \eta \leq \frac{1}{4}$ using the following inequality (see [4], p. 58)

$$
\left|\frac{\Gamma(1-s)}{\Gamma(s)}\right| \leq 1.4 \max \left(1,|s|^{1+2 \eta}\right)
$$

From (26) and (27) we obtain

$$
|g(-\eta+i t, \chi)| \leq 1.4^{r_{2}} A(\mathfrak{f})^{1+2 \eta}\left(\max \left(1,|-\eta+i t|^{(1+2 \eta)}\right)\right)^{r_{2}}
$$

for $-\infty<t<\infty$. Write

$$
G(s, \chi)=\frac{\zeta(s, \chi)}{(s+1)^{r_{2}(1+2 \eta)}} .
$$

From (28) we have

$$
\begin{aligned}
|G(-\eta+i t, \chi)| & \leq 1.4^{r_{2}} A(\mathfrak{f})^{1+2 \eta}|\zeta(1+\eta, \bar{\chi})| \\
& \leq 1.4^{r_{2}} A(\mathfrak{f})^{1+2 \eta} \zeta(1+\eta)^{2 r_{2}}
\end{aligned}
$$

for $\chi \neq \chi_{0}$. If $\chi$ is not a primitive character, then there is an ideal $\mathfrak{f}_{0}$ which divides $\mathfrak{f}$, and there is a primitive character $\psi\left(\bmod \mathfrak{f}_{0}\right)$ such that

$$
\zeta(s, \chi)=\zeta(s, \psi) \prod_{\mathfrak{p}|\mathfrak{f}, \mathfrak{p}| \mathfrak{f}_{0}}\left(1-\frac{\psi(\mathfrak{p})}{(N \mathfrak{p})^{s}}\right) .
$$


Write $\mathfrak{f}=\mathfrak{f}_{0} \mathfrak{f}_{1}$. From [4, see p. 60 ] we get

$$
\left|\prod_{\mathfrak{p}|\mathfrak{f}, \mathfrak{p}| \mathfrak{f}_{0}}\left(1-\frac{\psi(\mathfrak{p})}{(N \mathfrak{p})^{s}}\right)\right| \leq 2\left(N \mathfrak{f}_{1}\right)^{\frac{1}{2}+\eta}
$$

Hence,

$$
\begin{aligned}
|G(-\eta+i t, \chi)| & \leq 1.4^{r_{2}}\left(1+\varepsilon_{\chi}\right) A(\mathfrak{f})^{1+2 \eta}|\zeta(1+\eta, \bar{\chi})| \\
& \leq 1.4^{r_{2}} A(\mathfrak{f})^{1+2 \eta} \zeta(1+\eta)^{2 r_{2}}
\end{aligned}
$$

for any character $\chi \neq \chi_{0}$ modulo $\mathfrak{f}$, where $\varepsilon_{\chi}=0$ or 1 to accordingly whether $\chi$ is primitive or not. On the other hand,

$$
|G(3+i t, \chi)| \leq \frac{|\zeta(3+\eta, \bar{\chi})|}{(4+i t)^{r_{2}(1+2 \eta)}} \leq \frac{1}{4^{r_{2}}} \zeta(3+\eta)^{2 r_{2}} .
$$

Using the estimate

$$
|\zeta(s, \chi)| \leq A_{1} e^{A_{2}|t|},
$$

which is valid in the strip $-\eta \leq \sigma \leq 3$, where $A_{1}, A_{2}$ depends on $K$, $\chi$, and $\mathfrak{f}$, we get

$$
|G(s, \chi)|=O\left(e^{A_{3}|t|}\right)
$$

for $-\eta \leq \sigma \leq 3$. From (31)-(33) and the well-known theorem of PhragmenLindelöf we obtain

$$
|G(s, \chi)| \leq \frac{1.4^{r_{2}}\left(1+\varepsilon_{\chi}\right) A(\mathfrak{f})^{1+2 \eta} \zeta(1+\eta)^{2 r_{2}}}{(|t|+1)^{r_{2}(1+2 \eta)}}
$$

in the strip $-\eta \leq \sigma \leq 3$. From (34), (29)

$$
|\zeta(s, \chi)| \leq 1.4^{r_{2}}\left(1+\varepsilon_{\chi}\right) A(\mathfrak{f})^{1+2 \eta} \zeta(1+\eta)^{2 r_{2}}(|t|+1)^{r_{2}(1+2 \eta)}
$$

for any character $\chi \neq \chi_{0}$ modulo $\mathfrak{f}$, where $\varepsilon_{\chi}=0$ or 1 to accordingly whether $\chi$ is primitive or not. This finishes the proof.

Lemma 10. For $\sigma>1$ we have

$$
\frac{1}{|\zeta(\sigma+i t, \chi)|} \leq \zeta_{K}(\sigma)
$$

Proof. See [?, Lemma 2.4].

Lemma 11. Let $[K: \mathbb{Q}]=2 r_{2}$ and $0<\eta \leq \frac{1}{4}$. In the region $-\eta \leq \sigma \leq 1+\eta$, $-\infty<t<\infty$ we have estimate

$$
\left|(s-1) \zeta\left(s, \chi_{0}\right)\right| \leq(3+|t|)(1+|t|)^{r_{2}(1+\eta-\sigma)}(|\Delta| N \mathfrak{f})^{\frac{1+\eta-\sigma}{2}} \zeta_{K}(1+\eta) .
$$


Proof. See [4, (5.4)].

Lemma 12. Let $f(s)$ be a function regular in the circle $\left|s-s_{0}\right| \leq r$ and satisfying the in equality

$$
\left|\frac{f(s)}{f\left(s_{0}\right)}\right| \leq M
$$

If $f(s) \neq 0$ in the region $\left|s-s_{0}\right| \leq \frac{r}{2}, \Re\left(s-s_{0}\right)>0$, then

$$
\Re \frac{f^{\prime}}{f}\left(s_{0}\right) \geq-\frac{4}{r} \log M
$$

Proof. See [11, p. 384-385

Lemma 13. Let $f(s)$ be a function regular in the circle $\left|s-s_{0}\right| \leq R$ and satisfying the coditions

$$
\Re f(s) \leq M \quad \text { for } \quad\left|s-s_{0}\right|=R
$$

Then

$$
\left|f^{(k)}(s)\right| \leq 2 k !\left(M-\Re f\left(s_{0}\right)\right) \frac{R}{(R-r)^{k+1}}, \quad k \geq 1 .
$$

in the circle $\left|s-s_{0}\right| \leq r<R$.

Proof. See [1], p. 384-385

We are in a position to prove Lemma 1 .

Proof. Let $B=\frac{A_{1}}{6}=0.01325$, where $A_{1}$ is the constant appearing in (44). Let $s_{0}=\sigma_{0}+i t_{0}, t_{0} \geq 0$,

$$
\sigma_{0}=1+\frac{B}{L\left(t_{0}\right)}
$$

where $L\left(t_{0}\right)$ is defined in (3). We define the function

$$
H(s, \chi)=\log \frac{g(s, \chi)}{g\left(s_{0}, \chi\right)}, \quad g(s)=h(s, \chi) \prod_{\rho}(s-\rho)^{-1},
$$

where $h(s, \chi)=\zeta(s, \chi)$ if $\chi \neq \chi_{0}$ and $h\left(s, \chi_{0}\right)=(s-1) \zeta\left(s, \chi_{0}\right)$, where $\rho$ are zeros of the function $h(s, \chi)$ in the circle $\left|s-s_{0}\right| \leq \frac{1}{2}$. Firstly, we estimate $\left|\frac{g(s)}{g\left(s_{0}\right)}\right|$. Lemmas 9 10 and 11] shows that in the circle $\left|s-\sigma_{0}\right| \leq 1$

$$
\left|\frac{\zeta(\sigma+i t, \chi)}{\zeta\left(\sigma_{0}, \chi\right)}\right| \leq 1.4\left(1+\varepsilon_{\chi}\right) A(\mathfrak{f})^{1+2 \eta} \zeta(1+\eta)^{2 r_{2}} \zeta_{K}\left(\sigma_{0}\right)\left(t_{0}+2\right)^{r_{2}(1+2 \eta)},
$$


for any character $\chi \neq \chi_{0}$ modulo $\mathfrak{f}$, where $\varepsilon_{\chi}=0$ or 1 to accordingly whether $\chi$ is primitive or not, and

$$
\begin{aligned}
& \left|\frac{\zeta\left(\sigma+i t, \chi_{0}\right)(s-1)}{\zeta\left(\sigma_{0}, \chi_{0}\right)\left(\sigma_{0}-1\right)}\right| \leq \\
& \quad \leq \frac{L\left(t_{0}\right)}{B}\left(4+\left|t_{0}\right|\right)\left(2+\left|t_{0}\right|\right)^{r_{2}(1+\eta)}(|\Delta| N \mathfrak{f})^{\frac{1+\eta}{2}} \zeta_{K}(1+\eta) \zeta_{K}\left(\sigma_{0}\right) .
\end{aligned}
$$

On $\left|s-s_{0}\right|=1,\left|s_{0}-\rho\right| \leq \frac{1}{2}$ and $|s-\rho| \geq \frac{1}{2}$. From (36), (37) and the maximum principle we obtain

$$
\begin{aligned}
& \left|\frac{\zeta(s, \chi) \prod_{\rho}\left(s_{0}-\rho\right)}{\zeta\left(s_{0}, \chi\right) \prod_{\rho}(s-\rho)}\right| \leq \\
& \quad \leq 1.4\left(1+\varepsilon_{\chi}\right) A(\mathfrak{f})^{1+2 \eta} \zeta(1+\eta)^{2 r_{2}} \zeta_{K}\left(\sigma_{0}\right)\left(t_{0}+2\right)^{r_{2}(1+2 \eta)}
\end{aligned}
$$

and

$$
\begin{aligned}
& \left|\frac{(s-1) \zeta\left(s, \chi_{0}\right) \prod_{\rho}\left(s_{0}-\rho\right)}{\left(s_{0}-1\right) \zeta\left(s_{0}, \chi_{0}\right) \prod_{\rho}(s-\rho)}\right| \leq\left|\frac{(s-1) \zeta\left(s, \chi_{0}\right) \prod_{\rho}\left(s_{0}-\rho\right)}{\left(\sigma_{0}-1\right) \zeta\left(s_{0}, \chi_{0}\right) \prod_{\rho}(s-\rho)}\right| \\
& \leq \frac{L\left(t_{0}\right)}{B}\left(4+\left|t_{0}\right|\right)\left(2+\left|t_{0}\right|\right)^{r_{2}(1+\eta)}(|\Delta| N \mathfrak{f})^{\frac{1+\eta}{2}} \zeta_{K}(1+\eta) \zeta_{K}\left(\sigma_{0}\right)
\end{aligned}
$$

in the circle $\left|s-s_{0}\right| \leq 1$. Secondly, we apply Lemma 13 to the function $H(s, \chi)$ with $k=1, R=\frac{1}{2}$ and $r=\frac{1}{4}$. The function $H(s, \chi)$ is regular in the circle $\left|s-s_{0}\right| \leq \frac{1}{2}$, so by (38), (38) we obtain

$$
\begin{aligned}
& \Re H(s, \chi)=\log \left|\frac{g(s, \chi)}{g\left(s_{0}, \chi\right)}\right| \leq \\
& \quad \leq \begin{cases}\left.\log \left(1.4\left(1+\varepsilon_{\chi}\right) A(\mathfrak{f})^{1+2 \eta} \zeta(1+\eta)^{2 r_{2}} \zeta_{K}\left(\sigma_{0}\right)\left(t_{0}+2\right)^{r_{2}(1+2 \eta)}\right)\right), & \text { if } \chi \neq \chi_{0} \\
\log \left(\frac{L\left(t_{0}\right)}{B}\left(4+\left|t_{0}\right|\right)\left(2+\left|t_{0}\right|\right)^{r_{2}(1+\eta)}(|\Delta| N \mathfrak{f})^{\frac{1+\eta}{2}} \zeta_{K}(1+\eta) \zeta_{K}\left(\sigma_{0}\right)\right), & \text { if } \chi=\chi_{0} .\end{cases}
\end{aligned}
$$

in the circle $\left|s-s_{0}\right| \leq \frac{1}{2}$. Therefore, in the circle $\left|s-s_{0}\right| \leq \frac{1}{4}$ we have

$$
\begin{aligned}
\mid \frac{\zeta^{\prime}}{\zeta}(s, \chi) & -\sum_{\rho} \frac{1}{s-\rho} \mid \leq \\
& \leq 16 \log \left(1.4\left(1+\varepsilon_{\chi}\right) A(\mathfrak{f})^{1+2 \eta} \zeta(1+\eta)^{2 r_{2}} \zeta_{K}\left(\sigma_{0}\right)\left(t_{0}+2\right)^{r_{2}(1+2 \eta)}\right)
\end{aligned}
$$

and

$$
\begin{aligned}
\mid \frac{\zeta^{\prime}}{\zeta}\left(s, \chi_{0}\right) & +\frac{1}{s-1}-\sum_{\rho} \frac{1}{s-\rho} \mid \leq \\
\leq & 16 \log \left(\frac{L\left(t_{0}\right)}{B}\left(4+\left|t_{0}\right|\right)\left(2+\left|t_{0}\right|\right)^{r_{2}(1+\eta)}(|\Delta| N \mathfrak{f})^{\frac{1+\eta}{2}} \zeta_{K}(1+\eta) \zeta_{K}\left(\sigma_{0}\right)\right) .
\end{aligned}
$$


Finally, we estimate $\left|\sum_{\rho} \frac{1}{s_{0}-\rho}\right|$ and $\left|\sum_{\rho} \frac{1}{s-\rho}\right|$. In [10] Israilov show that, if $1<$ $\sigma \leq 2$ then

$$
-\frac{\zeta^{\prime}}{\zeta}(\sigma)<\frac{1}{\sigma-1}-\gamma+C_{1}(\sigma-1)
$$

where $C_{1}=0.1875463$. Hence, (35) shows

$$
\left|\frac{\zeta^{\prime}}{\zeta}\left(s_{0}, \chi\right)\right| \leq 2 r_{2} \frac{L\left(t_{0}\right)}{B}+2 r_{2} C_{1} \frac{B}{L\left(t_{0}\right)}
$$

and

$$
\left|\frac{\zeta^{\prime}}{\zeta}\left(s_{0}, \chi_{0}\right)+\frac{1}{s_{0}-1}\right| \leq 4 r_{2} \frac{L\left(t_{0}\right)}{B}+2 r_{2} C_{1} \frac{B}{L\left(t_{0}\right)} .
$$

By (40), (42), we obtain

$$
\begin{aligned}
\left|\sum_{\rho} \frac{1}{s_{0}-\rho}\right| & \left.\leq 16 \log \left(1.4\left(1+\varepsilon_{\chi}\right) A(\mathfrak{f})^{1+2 \eta} \zeta(1+\eta)^{2 r_{2}} \zeta_{K}\left(\sigma_{0}\right)\left(t_{0}+2\right)^{r_{2}(1+2 \eta)}\right)\right) \\
& +2 r_{2} \frac{L\left(t_{0}\right)}{B}+2 r_{2} C_{1} \frac{B}{L\left(t_{0}\right)},
\end{aligned}
$$

and (41), (43)

$$
\begin{aligned}
\left|\sum_{\rho} \frac{1}{s_{0}-\rho}\right| & \leq 16 \log \left(\frac{L\left(t_{0}\right)}{B}\left(4+\left|t_{0}\right|\right)\left(2+\left|t_{0}\right|\right)^{r_{2}(1+\eta)}(|\Delta| N \mathfrak{f})^{\frac{1+\eta}{2}} \zeta_{K}(1+\eta) \zeta_{K}\left(\sigma_{0}\right)\right) \\
& +4 r_{2} \frac{L\left(t_{0}\right)}{B}+2 r_{2} C_{1} \frac{B}{L\left(t_{0}\right)} .
\end{aligned}
$$

in the circle $\left|s-s_{0}\right| \leq \frac{1}{4}$. Now, we define

$$
r_{1}=\frac{2 B}{L\left(t_{0}\right)}<\frac{1}{4}
$$

By Theorem 3, the function $\zeta(s, \chi) \neq 0$ in the region $\left|s-s_{0}\right| \leq r, \Re\left(s-s_{0}\right)>$ $-2 r_{1}$. Hence

$$
\left|s_{0}-\rho\right| \geq 2 r_{1}, \quad|s-\rho| \geq \frac{1}{2}\left|s_{0}-\rho\right|, \quad \Re\left(s_{0}-\rho\right) \geq 2 r_{1}
$$

for all zeros $\rho$ in the circle $\left|s-s_{0}\right| \leq \frac{1}{4}$, and for $s$ in the circle $\left|s-s_{0}\right| \leq r_{1}$. For $\left|s-s_{0}\right| \leq r_{1}$ we obtain

$$
\begin{aligned}
\left|\sum_{\rho} \frac{1}{s-\rho}-\sum_{\rho} \frac{1}{s_{0}-\rho}\right| & \leq \sum_{\rho} \frac{\left|s-s_{0}\right|}{|s-\rho|\left|s_{0}-\rho\right|} \leq \sum_{\rho} \frac{r_{1}}{\frac{1}{2}\left|s_{0}-\rho\right|^{2}} \\
& \leq \sum_{\rho} \frac{\Re\left(s_{0}-\rho\right)}{\left|s_{0}-\rho\right|^{2}} \leq \sum_{\rho} \Re \frac{1}{s_{0}-\rho} \leq\left|\sum_{\rho} \frac{1}{s_{0}-\rho}\right| .
\end{aligned}
$$


Thus,

$$
\left|\sum_{\rho} \frac{1}{s-\rho}\right| \leq 2\left|\sum_{\rho} \frac{1}{s_{0}-\rho}\right|
$$

From (40), (44) and (46) we have

$$
\begin{aligned}
\left|\frac{\zeta^{\prime}}{\zeta}(s, \chi)\right| & \leq 4 r_{2} \frac{L\left(t_{0}\right)}{B}+4 r_{2} C_{1} \frac{B}{L\left(t_{0}\right)} \\
& +32 \log \left(1.4\left(1+\varepsilon_{\chi}\right) A(\mathfrak{f})^{1+2 \eta} \zeta(1+\eta)^{2 r_{2}} \zeta_{K}\left(\sigma_{0}\right)\left(t_{0}+2\right)^{r_{2}(1+2 \eta)}\right)
\end{aligned}
$$

and by (40), 45) and (46)

$$
\begin{aligned}
\left|\frac{\zeta^{\prime}}{\zeta}(s, \chi)+\frac{1}{s-1}\right| & \leq 8 r_{2} \frac{L\left(t_{0}\right)}{B}+4 r_{2} C_{1} \frac{B}{L\left(t_{0}\right)} \\
& +32 \log \left(\frac{L\left(t_{0}\right)}{B}\left(4+\left|t_{0}\right|\right)\left(2+\left|t_{0}\right|\right)^{r_{2}(1+\eta)}(|\Delta| N \mathfrak{f})^{\frac{1+\eta}{2}} \zeta_{K}(1+\eta) \zeta_{K}\left(\sigma_{0}\right)\right)
\end{aligned}
$$

in the circle $\left|s-s_{0}\right| \leq r_{1}$, and consequently in the strip

$$
1-\frac{B}{L(t)}=1-\frac{A_{1}}{6 L(t)}<\sigma<1+\frac{3 B}{L(t)}=1+\frac{A_{1}}{2 L(t)}
$$

Put $s_{0}=\sigma_{0}+t_{0}, t_{0} \geq 0$, and

$$
1+\frac{A_{1}}{2 L\left(t_{0}\right)} \leq \sigma \leq 3
$$

Lemma 12 and (36), (37) shows that

$$
-\frac{\zeta^{\prime}}{\zeta}\left(\sigma_{0}, \chi\right) \leq 4 \log \left(1.4\left(1+\varepsilon_{\chi}\right) A(\mathfrak{f})^{1+2 \eta} \zeta(1+\eta)^{2 r_{2}} \zeta_{K}\left(\sigma_{0}\right)\left(t_{0}+2\right)^{r_{2}(1+2 \eta)}\right)
$$

for any character $\chi \neq \chi_{0}$ modulo $\mathfrak{f}$, where $\varepsilon_{\chi}=0$ or 1 to accordingly whether $\chi$ is primitive or not, and

$$
\begin{aligned}
-\frac{\zeta^{\prime}}{\zeta}\left(\sigma_{0}, \chi_{0}\right) & \leq \frac{1}{\sigma_{0}-1}+ \\
& +4 \log \left(\frac{L\left(t_{0}\right)}{B}\left(4+\left|t_{0}\right|\right)\left(2+\left|t_{0}\right|\right)^{r_{2}(1+\eta)}(|\Delta| N \mathfrak{f})^{\frac{1+\eta}{2}} \zeta_{K}(1+\eta) \zeta_{K}\left(\sigma_{0}\right)\right)
\end{aligned}
$$

Therefore,

$$
\left|\frac{\zeta^{\prime}}{\zeta}\left(\sigma_{0}, \chi\right)\right| \leq 4 \log \left(1.4\left(1+\varepsilon_{\chi}\right) A(\mathfrak{f})^{1+2 \eta} \zeta(1+\eta)^{2 r_{2}} \zeta_{K}\left(\sigma_{0}\right)\left(t_{0}+2\right)^{r_{2}(1+2 \eta)}\right)
$$


for any character $\chi \neq \chi_{0}$ modulo $\mathfrak{f}$, where $\varepsilon_{\chi}=0$ or 1 to accordingly whether $\chi$ is primitive or not, and

$$
\begin{aligned}
\left|\frac{\zeta^{\prime}}{\zeta}\left(\sigma_{0}, \chi_{0}\right)\right| & \leq \frac{2 L\left(t_{0}\right)}{A_{1}}+ \\
& +4 \log \left(\frac{L\left(t_{0}\right)}{B}\left(4+\left|t_{0}\right|\right)\left(2+\left|t_{0}\right|\right)^{r_{2}(1+\eta)}(|\Delta| N \mathfrak{f})^{\frac{1+\eta}{2}} \zeta_{K}(1+\eta) \zeta_{K}\left(\sigma_{0}\right)\right) .
\end{aligned}
$$

By the above we obtain

$$
\begin{aligned}
\left|\frac{\zeta^{\prime}}{\zeta}\left(s_{0}, \chi\right)\right| & \leq\left|\frac{\zeta^{\prime}}{\zeta}\left(\sigma_{0}, \chi\right)\right| \leq \\
& 4 \log \left(1.4\left(1+\varepsilon_{\chi}\right) A(\mathfrak{f})^{1+2 \eta} \zeta(1+\eta)^{2 r_{2}} \zeta_{K}\left(\sigma_{0}\right)\left(t_{0}+2\right)^{r_{2}(1+2 \eta)}\right)
\end{aligned}
$$

for any character $\chi \neq \chi_{0}$ modulo $\mathfrak{f}$, where $\varepsilon_{\chi}=0$ or 1 to accordingly whether $\chi$ is primitive or not, and

$$
\begin{aligned}
\mid \frac{\zeta^{\prime}}{\zeta}\left(s_{0}, \chi_{0}\right) & +\frac{1}{s_{0}-1}|\leq| \frac{\zeta^{\prime}}{\zeta}\left(\sigma_{0}, \chi\right) \mid+\frac{1}{\sigma_{0}-1} \leq \frac{4 L\left(t_{0}\right)}{A_{1}}+ \\
& +4 \log \left(\frac{L\left(t_{0}\right)}{B}\left(4+\left|t_{0}\right|\right)\left(2+\left|t_{0}\right|\right)^{r_{2}(1+\eta)}(|\Delta| N \mathfrak{f})^{\frac{1+\eta}{2}} \zeta_{K}(1+\eta) \zeta_{K}\left(\sigma_{0}\right)\right) .
\end{aligned}
$$

The proof is completed by applying

$$
\zeta_{K}\left(\sigma_{0}\right) \leq \zeta\left(\sigma_{0}\right)^{2 r_{2}} \leq\left(1+\frac{6 L\left(t_{0}\right)}{A_{1}}\right)^{2 r_{2}} .
$$

\section{References}

[1] Atkin, A., Morain, F.: Elliptic curves and primality proving. Tech. rep., Projet ICSLA RR-1256, INRIA (1990)

[2] Chatzigeorgiou, I.: Bounds on the Lambert function and their application to the outage analysis of user cooperation. IEEE Communications Letters 17(7)

[3] Dupont, R., Enge, A., Morain, F.: Building curves with arbitrary small mov degree over finite prime fields. J. Cryptology 18(2), 79-89 (2005)

[4] Fryska, T.: An estimate of the order of the hecke-landau $\zeta(s, \chi)$-functions. Funct. Approx. Comment. Math. 16, 55-62 (1988)

[5] Fryska, T.: Some effective estimates for the roots of the dirichlet l-series, ii. Funct. Approx. Comment. Math. 16, 21-36 (1988)

[6] Grześkowiak, M.: An algorithmic construction of finite elliptic curves of order divisible by a large prime. Fund. Inform.

[7] Grześkowiak, M.: Algorithms for relatively cyclotomic primes. Fund. Inform. 125(2), 161-181 (2013)

[8] Grześkowiak, M.: Algorithms for pairing-friendly primes. In: Pairing-Based Cryptography 2013, LNCS 8365. pp. 215-288 (2014)

[9] Ingham, A.E.: The Distribution of Prime Numbers. Univ. Press Cambridge (1932)

[10] Israilov, M.: The Laurent expansion of the Riemann zeta function. Trudy Mat. Inst. Steklov. 158, 98-104 (1981)

[11] Prachar, K.: Primzahlverteilung. Berlin (1957)

[12] Radziejewski, M.: http://www.staff.amu.edu.pl/m̃aciejr 